\title{
Mobilité en site propre et forme urbaine : une possible interaction?
}

\section{Anne Hecker}

\section{Q OpenEdition}

\section{Journals}

Édition électronique

URL : http://journals.openedition.org/rge/3623

DOI : $10.4000 /$ rge.3623

ISSN : 2108-6478

Éditeur

Association des géographes de l'Est

Édition imprimée

Date de publication : 15 juin 2012

ISSN : 0035-3213

\section{Référence électronique}

Anne Hecker, « Mobilité en site propre et forme urbaine : une possible interaction ? », Revue

Géographique de l'Est [En ligne], vol. 52 / 1-2 | 2012, mis en ligne le 04 février 2013, consulté le 08 septembre 2020. URL : http://journals.openedition.org/rge/3623 ; DOI : https://doi.org/10.4000/rge. 3623

Ce document a été généré automatiquement le 8 septembre 2020.

Tous droits réservés 


\title{
Mobilité en site propre et forme urbaine : une possible interaction?
}

\author{
Anne Hecker
}

« Il y a évidemment un paradoxe entre la dénonciation permanente de l'étalement urbain (presque unanime chez les professionnels), alors que dans le même temps, sur le terrain, il ne semble jamais s'être aussi bien porté » (Wiel 2010).

1 La ville française de ce début de XXIè siècle se voudrait « durable ", mixte et compacte, en réponse aux craintes diffuses du peak oil ${ }^{1}$ et des pollutions, à la menace des congestions et aux difficultés sociales nées de la périurbanisation. Elle n'en demeure pas moins encore diffuse et dépendante d'(auto)mobilités croissantes, chaînées et lointaines. Ces banlieues étalées, polynucléaires mais monofonctionnelles, ont été majoritairement conçues autour des infrastructures de transport routières. Leur étalement, longtemps jugé inévitable et mal maîtrisé, n'a pas rendu possible leur desserte efficace par les transports en commun, particulièrement par les Transports Collectifs en Site Propre (TCSP*)2. Elles présentent pourtant de forts enjeux dans le domaine des mobilités. A contrario le cœur historique, dense en population comme en activités, conçu à l'échelle du piéton, est efficacement desservi par les transports en commun. Mixte économiquement et fonctionnellement, porteur des valeurs d'urbanité, il est pourtant fui par une partie de la population, de même que la première couronne, déjà moins dense mais encore très urbaine et toujours bien desservie, mais peu recherchée au regard de la grande banlieue (Kaufmann et Jemelin 2003).

2 Ces paradoxes et l'idée d'un désir fort de périurbanisation attribué à la population urbaine (Mancebo 2008) ont contribué à freiner en France la lutte contre l'étalement urbain, avant que ne s'installe un consensus, désormais traduit dans la législation. La volonté d'une cohérence entre l'urbanisme, les transports et l'habitat est annoncée dans la loi SRU*, tandis que la nécessaire articulation des mesures sectorielles, gérées à des échelles urbaines fonctionnelles, s'affirme avec force dans la dernière « loi portant engagement national pour l'environnement» (2010 - $788 \mathrm{du} 12$ janvier 2010) dite «Grenelle 2 ». Le lien entre forme urbaine et transports en commun, particulièrement en site propre, devient l'une des priorités des documents d'urbanisme. Mais des mesures législatives, même adossées à ces documents d'aménagement qui ont montré 
leurs limites par le passé (Mancebo 2008), peuvent-elles être rapidement efficaces face à un modèle urbain français constitué depuis un demi-siècle autour de l'automobilité et de banlieues périurbaines mal desservies par les TCSP* ? Ou peut-on craindre un effet d'annonce, contrarié par une difficile mise en œuvre?

L'Allemagne s'est lancée depuis les années 1990 dans de telles politiques avec un certain succès qu'il est possible de convier pour mieux appréhender le cas français. Sans tomber dans l'écueil de la transférabilité potentielle d'un modèle, l'analyse comparée de ces deux systèmes urbains permet de pointer leurs avancées et les blocages qu'ils subissent. Il conviendra d'évoquer des contextes structurels et politiques différents, à l'origine de mises en pratique diversifiées de l'articulation urbanisme-TCSP*. Mais audelà de ces différences fondamentales, le bilan comparé d'une quinzaine d'années d'expérimentation dans les agglomérations de Münster et de Toulouse illustrera les rôles différents attribués aux TCSP* en Allemagne et en France. On notera toutefois que l'inflexion du contraignant modèle allemand, ainsi que la mise en œuvre des contrats d'axe français pourraient faire converger ces deux modèles vers une plus grande uniformité de la coordination des politiques sectorielles, au service d'une meilleure articulation transports en commun/ forme urbaine.

Pourquoi Toulouse et Münster?

Le choix des deux communes de Münster et de Toulouse résulte d'une double décision : d'une part celle de sélectionner deux agglomérations suffisamment représentatives des structures, des politiques, des forces et des faiblesses de leur État respectif pour éviter l'écueil de l'exemple atypique et autoriser l'élargissement de la réflexion au-delà du cas particulier ; d'autre part celle de comparer deux espaces comparables par la taille, les réseaux de transport collectif, la volonté affichée de lutter contre l'étalement urbain, et présentant depuis les premières mesures un recul comparable et suffisant pour en extraire quelques premiers éléments de bilan.

\begin{tabular}{|c|c|c|}
\hline & Münster & Toulouse \\
\hline $\begin{array}{l}\text { Taille du bassin } \\
\text { de vie } \\
\text { centre) }\end{array}$ & $\begin{array}{l}1,5 \text { million habitants } \\
(280000)\end{array}$ & $\begin{array}{l}\begin{array}{l}1,2 \\
(439\end{array} \text { 000) }\end{array}$ \\
\hline $\begin{array}{l}\text { Réseaux de TCSP } \\
\text { desservant } 1 \text { Iè } \\
\text { couronne }\end{array}$ & $\begin{array}{l}9 \text { lignes de S-Bahn - } \\
\text { dense réseau de bus }\end{array}$ & $\begin{array}{l}2 \text { lignes de métro, } 1 \text { de } \\
\text { tramway, } 1 \text { voie ferrée } \\
\text { requalifiée en RER, } 2 \text { TCSP } \\
\text { - dense réseau de bus }\end{array}$ \\
\hline $\begin{array}{ll}\text { Mesures } & d e \\
\text { subordination } & d e \\
\text { l'urbanisation } & \\
\text { aux TCSP } & \end{array}$ & $\begin{array}{l}\text { Mesures d'orientation / } \\
\text { blocage de } \\
\text { l'urbanisation selon } \\
\text { éloignement des TCSP / } \\
\text { gares; achat prospectif } \\
\text { de terrains bien placés }\end{array}$ & $\begin{array}{l}\text { Préconisations de liens } \\
\text { urbanisme / TCSP ; } 4 \text { SCoT } \\
\text { en cours d'élaboration, } \\
\text { une démarche Inter-SCoT; } \\
\text { projets de multiples } \\
\text { contrats d'axe }\end{array}$ \\
\hline
\end{tabular}




\begin{tabular}{|l|ll|l|}
\hline $\begin{array}{l}\text { Date des } \\
\text { premières } \\
\text { mesures }\end{array}$ & $\begin{array}{l}1990, \\
1995\end{array}$ & renforcées en & 1995 \\
\hline
\end{tabular}

\section{Des contextes différents au service d'une même idée : subordonner l'urbanisme aux transports collectifs}

6 La France et l'Allemagne se trouvent confrontées à l'artificialisation galopante de leur territoire, au même attrait (choisi ou contraint) de la population pour la maison individuelle, à l'augmentation des superficies des logements et à une consommation accrue d'espace par emploi. Cette pression foncière sur les grandes agglomérations amène de fait une dispersion dans le périurbain, placée sous le symbole de l'automobile et d'une structure monofonctionnelle dominantes. Dès lors, elles ont affiché dans leurs législations respectives une volonté identique de mieux coordonner leur forme urbaine aux transports collectifs les plus efficients.

7 En France, cet engagement ne s'impose qu'assez récemment. Les premières mesures sur les mobilités urbaines apparaissent avec la LOTI* de 1982, mais ce n'est qu'en 1996 avec la LAURE* que le Plan de Déplacement Urbain (PDU) est rendu obligatoire pour les agglomérations de plus de 100000 habitants. L'idée de mise en cohérence des politiques sectorielles dans les domaines des transports et de l'urbanisme naît avec la loi SRU* en 2000. Elle modifie l'article 121-1 du Code de l'Urbanisme pour imposer «un développement urbain maitrisé » et « une utilisation économe et équilibrée des espaces naturels, urbains, périurbains et ruraux, la maîtrise des besoins de déplacement et de la circulation automobile (...) ». Les nouveaux documents de planification qu'elle crée doivent favoriser l'articulation urbanisme / transports, notamment les Schémas de cohérence Territoriale (SCoT) dont le rôle est confirmé dans les Lois dites "Grenelle». La deuxième du nom évoque clairement la soumission de l'urbanisation aux transports collectifs. Elle amende à nouveau le Code de l'urbanisme (article 122-1) pour préciser que les SCoT* "définissent notamment les objectifs relatifs [...] à l'équilibre entre l'urbanisation et la création de dessertes en transports collectifs (...). Ils précisent les conditions permettant de favoriser le développement de l'urbanisation prioritaire dans les secteurs desservis par les transports collectifs. Ils peuvent, le cas échéant, subordonner l'ouverture à l'urbanisation de zones naturelles ou agricoles et les extensions urbaines à la création de dessertes en transports collectifs et à l'utilisation préalable de terrains situés en zone urbanisée et desservis par les équipements $»^{3}$.

8 La France fonde donc sur le $\mathrm{SCoT}^{*}$ ses espoirs de subordination de l'urbanisme aux transports collectifs. Il a le mérite d'être opposable aux autres documents d'urbanisme (notamment PLU et $\mathrm{PDU}^{4}$ ) et de s'appuyer sur un périmètre défini à l'échelle fonctionnelle d'un bassin de vie ou d'une aire urbaine. Il ajoute toutefois un échelon supplémentaire à une structure d'aménagement déjà complexe (voir infra) aux périmètres parfois incohérents. Par ailleurs, sa mise en œuvre est longue : 9 ans après

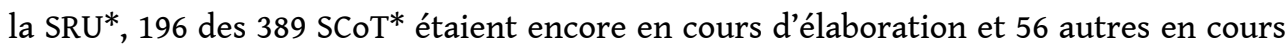
d'approbation ${ }^{5} .$. au risque de voir évoluer la périurbanisation au-delà de leur 
périmètre avant leur finalisation. Enfin, près de la moitié d'entre eux n'intégreront les nouvelles dispositions du Code de l'Urbanisme amendé par le Grenelle 2 que lors de leur future révision, retardant d'autant la réelle application de ces principes. On peut également regretter que dans les plus grandes agglomérations comme Toulouse, la taille modérée des $\mathrm{SCoT}^{*}$ ne corresponde pas à la réalité de l'étalement urbain. Devant le manque de prise en compte de l'échelle métropolitaine dans sa globalité, les principaux acteurs de la planification locale - État, Présidents de SCoT* centraux, agences d'urbanisme - jugent nécessaire dès 2000 de coordonner l'action de ces nouveaux documents de planification. Ainsi, en 2011, cent-huit SCoT* ont entrepris une démarche de regroupement en 16 Inter-SCoT représentant l'essentiel des grandes agglomérations françaises. Cette démarche interterritoriale permet d'apporter la cohérence d'une vision globale à l'échelle de l'agglomération - par exemple aux onze SCoT* $^{*}$ se partageant l'aire métropolitaine lyonnaise - mais ajoute un échelon supplémentaire à la prise de décision.

9 Face à cette lente mise en œuvre, l'Allemagne semble avoir pris de l'avance. Elle bénéficie depuis le droit territorial prussien et les lois qui en découlent d'une forte tradition aménagiste. Elle s'est notamment traduite par le maintien des réseaux de tramways dans la plupart des grandes villes et le rôle de principal outil de déplacement urbain qui leur est attribué lors de la reconstruction.En 1965, la Loi sur l'Aménagement du territoire (Raumordnungsgesetz) énonçait déjà l'idée de contenir l'extension spatiale le long d'axes de développement, structurés par les voies de communication principales reliant entre eux des lieux centraux bien équipés. Régulièrement, ce principe a été réaffirmé depuis, notamment dans le code de la construction (Baugesetzbuch), qui constitue le cadre juridique de la planification urbaine en Allemagne. Si la « durabilité » de la ville apparaît en filigrane dans l'aide au développement urbain (Code fédéral de la construction, 1971) c'est dans les années 1990 que l'idée de pérennité du développement s'impose en tant que concept intégrateur de la politique urbaine. La réunification et les craintes de pression migratoire attendue dans certaines villes ouestallemandes renouvellent le cadre du développement urbain et débouchent sur la mise en œuvre de nouveaux concepts. L'État évoque alors dans un rapport ${ }^{6} l^{1}$ objectif d'une " ville compacte et multifonctionnelle dans des régions poly-concentriques ", et introduit dans la Loi fédérale sur l'aménagement du territoire le concept de "concentration décentralisée ». Issue de la théorie des lieux centraux, elle conforte la structure urbaine multipolaire hiérarchisée par des décennies de planification, et détourne l'étalement urbain des marges rurales pour le contraindre vers les pôles secondaires des agglomérations. Densités urbaines, «ville des courtes distances » multifonctionnelles, et jonction des pôles par les réseaux de transports collectifs, notamment ferroviaires, sont les priorités de ce concept.

10 Les villes allemandes s'intègrent dans un mouvement de rétrécissement urbain, débuté précocement et dans le contexte socio-politique spécifique de l'après-réunification dans les nouveaux Länder (Schrumpfende Städte). La décroissance démographique atteint aujourd'hui l'ensemble du pays - 26 villes de plus de 100000 habitants sont concernées en Allemagne. Alors que l'on pourrait s'attendre à une atténuation globale de la pression à l'étalement, les appellations évoquant le rétrécissement sont souvent trompeuses, les villes supposées " rétrécir " s'inscrivant a contrario dans des agglomérations qui poursuivent leur étalement (Fol et Cunningham-Sabot 2010). Dans ce contexte, certaines communes-relais allemandes sont ciblées pour grandir et concentrer population, emplois et équipements, particulièrement celles bénéficiant 
d'une desserte en transports ferroviaires de type RER* (S-Bahn). En effet, la concentration décentralisée insiste également sur une offre urbaine "aux bons endroits", devenue principe fédéral: toute nouvelle extension urbaine doit être implantée prioritairement à proximité immédiate de dessertes ferroviaires ou de TCSP*; l'installation de lotissements dans des espaces isolés, desservis uniquement par le mode routier, doit a contrario être strictement limitée. Ce principe est favorisé par la bonne densité des banlieues allemandes, qui proposent davantage de maisons semicollectives en bandes que de pavillons individuels - plus de $60 \%$ de la population vit dans un bâtiment multifamilial, contre 40 \% en France (Kauffmann et Jemelin 2003). Elles sont en outre bien desservies par un réseau ferré multipliant les liaisons radiales, et constituent ainsi une opportunité d'y orienter la population désireuse de s'éloigner du centre-ville, et de subordonner l'urbanisme aux transports collectifs.

11 Autre facteur facilitant, la réforme administrative des années 1960 (achevée à la réunification) à l'origine d'un vaste processus de fusion communale : l'Allemagne ne compte plus aujourd'hui que 12291 communes, d'une taille moyenne proche des 6000 habitants - contre 2000 en France - au pouvoir renforcé. Les plus importantes (généralement supérieures à 100000 habitants) bénéficient en outre du statut d'arrondissement (Kreis), cumul qui supprime un échelon administratif et renforce leur pouvoir décisionnel quant à l'aménagement de leur territoire. Elles s'intègrent en effet dans une structure articuléeen trois niveaux juridiquement autonomes (Fédération, Land et commune), auxquels s'ajoute l'arrondissement pour les petites communes entité supra-communale sans compétence juridique en termes de planification, mais responsable dans son périmètre de l'investissement et du fonctionnement des services collectifs tels que les transports publics (Jouve 2003). Dans ce cadre, la planification spatiale bénéficie d'une forte décentralisation : la Fédération publie les lois générales, le code de l'urbanisme ainsi que la planification générale des déplacements ; le Land décline ces orientations législatives générales dans ses propres lois, notamment les plans généraux de déplacement et de développement urbain, documents de référence que les arrondissements et les communes déclinent sur leurs propres périmètres. La planification urbaine locale, les plans de construction, ceux de déplacement locaux et la localisation des infrastructures relèvent directement de l'autorité communale (OCDE 1999). En matière d'autorités organisatrices de transports (AOT*), l'Allemagne se caractérise par la séparation des modes: les transports ferroviaires régionaux sont confiés au Land, qui planifie et constitue l'offre de service envisagées, et sous-traite la gestion à l'opérateur historique - la DB - ou à un tout autre opérateur international depuis l'ouverture à la concurrence. Le Land peut également créer des communautés de transport, notamment tarifaires.Quant à la gestion des transports urbains, elle relève de l'autorité des Kreise ou directement des communes-arrondissements à l'intérieur de leur périmètre.

De cette simplicité - relative - émane une bonne cohérence des périmètres d'intervention en matière de planification urbaine et de transports, surtout au regard de la situation française. État, Région, Département, commune, intercommunalité, pays, agglomération, aire et unité urbaine, district, $\mathrm{SCoT}^{*}$, Périmètre des Transports Urbains (PTU) ... la multiplicité des acteurs et des échelles de décision, aux enjeux et territoires variés, nuit à l'efficience des projets. Ainsi, comment coordonner efficacement urbanisme et transports collectifs, quand la planification urbaine et la maîtrise des réseaux, des horaires ou des fréquences relèvent de structures différentes qui n'évoluent pas dans les mêmes périmètres? Toulouse en offre un bon exemple : elle 
présente en échelles emboîtées une ville (439000 habitants en 2007), une agglomération de 72 communes (858000 habitants), et une aire urbaine de 342 communes ( 1,1 millions d'habitants en $2007^{7}$ scindés en 5 départements et 2 régions). Dix-neuf intercommunalités (dont 3 majeures), recoupent ce premier découpage sans le recouvrir exactement. Par ailleurs, la Grande Agglomération Toulousaine se redécoupe en une communauté urbaine, 2 communautés d'agglomérations et 4 communautés de communes... L'unification des projets d'aménagement devrait découler du SCoT*, ou plutôt des $4 \mathrm{SCoT}^{*}$ qui se partagent cet immense territoire, regroupés depuis 2006 en un interSCoT de 433 communes pour plus d'un million d'habitants sur trois départements et deux régions administratives. Ils ne recouvrent toutefois pas totalement les périmètres précédents (notamment vingt communes de moins que la nouvelle aire urbaine), et s'ils sont opposables au PDU*, les périmètres manquaient là encore de cohérence : jusqu'à sa révision, $\mathrm{PDU}^{*}, \mathrm{SCoT}^{*}$ de la Grande Agglomération Toulousaine et transports urbains évoluaient dans des limites différentes.

13 Le cadre administratif, l'antériorité des mesures et le contexte général semblent donc favoriser l'Allemagne dans la volonté d'articuler transports collectifs et urbanisme. Le rôle accordé aux TCSP* ne peut que renforcer cette impression.

\section{Transports collectifs en site propre: desserte ou aménagement ?}

14 La justification première des lourds investissements dédiés aux transports en commun réside dans leur capacité à transporter les passagers. Néanmoins, on leur accorde aussi un rôle dans l'aménagement du territoire : beaucoup d'agglomérations ayant réalisé un TCSP* en ont fait le fil conducteur d'un projet de recomposition de l'espace urbain (Wiel 2010). La Loi dite Grenelle 2 impose ainsi aux SCoT* de définir «le développement de l'urbanisation prioritaire dans les secteurs desservis par les transports collectifs ».

Néanmoins, les projets français demeurent attachés en priorité à la desserte de la population, comme l'illustrent les récents réseaux de tramway. La desserte des polarités urbaines concentrant activités et équipements métropolitains (centres hospitaliers et universitaires, équipements culturels et sportifs, grands centres commerciaux ...) prime sur la liaison directe ${ }^{8}$. Celle du tissu urbain aux fortes densités de population permet «de capter dans un rayon théorique de $400 \mathrm{~m}$. autour des stations le maximum d'habitants potentiels possibles" (Laisney 2011). Ces choix traduisent la recherche d'une fréquentation digne de justifier les investissements consentis, au risque de cantonner les TCSP* aux métropoles denses et compactes, à l'étalement contrôlé le long de corridors d'urbanisation, et de disqualifier le périurbain diffus, ce car-dependant land use pattern de Newman et Kenworthy (1998). L'éloignement du centre (30 mn ou $10 \mathrm{~km}$ en moyenne) et l'entrée dans le trop peu dense habitat pavillonnaire compromettraient leur efficience.

16 L'exemple toulousain en est le reflet, dans le cadre d'une périurbanisation qui ne s'emballe pourtant que tardivement. A partir de la fin des années 1960, l'étalement se dilue dans un espace diffus, territoire de l'automobile triomphante, face à "l'indifférence souvent complète de l'offre de transports collectifs » (Marconis 2011), ou à son inadaptation aux nouveaux besoins de mobilité des périurbains. Il s'amplifie pourtant au moment de la création du syndicat mixte de transports collectifs $(1973,53$ communes, future 
AOT*), qui élargit la réflexion à l'échelle de la banlieue. Mais loin de coordonner l'urbanisation croissante à l'offre en cours de refonte, priorité est donnée au centre saturé et à sa traversée en souterrain. Le réseau actuel se structure donc autour de deux lignes de métro - VAL* (1993 et 2007), qui desservent la zone de forte densité de la ville-centre. S'y articulent une ligne de tramway vers Blagnac (2010), et une ligne de chemin de fer desservant l'ouest de l'agglomération, restructurée en 2003 en une desserte de type RER* et intégrée au réseau urbain. La vaste banlieue est desservie par un réseau de bus avec rabattement vers les terminus de métro ou du tramway.

17 Le principe de cohérence urbanisme-transport est pourtant mis en avant dès le projet d'agglomération de 1995, repris dans le Schéma Directeur de l'Agglomération Toulousaine de 1998. Il préconise une intensification urbaine le long des axes de transports collectifs, et le conditionnement de l'ouverture à l'urbanisation des larges territoires à la réalisation des infrastructures de transport nécessaires à leur fonctionnement. Vingt ans après ces premières mesures, la visualisation de l'avancée de l'artificialisation autour de Toulouse entre 1990 et 2006 que permet le Corine Land Cover ne délivre pas de résultats flagrants de l'efficacité de ces mesures (Figure 1). Les constructions nouvelles, qu'elles soient de nature résidentielle ou industrialocommerciale, s'éloignent des couronnes bien desservies par les transports collectifs en site propre. En effet, la densité des espaces desservis par ces courtes liaisons de TCSP* laisse peu de place à des opérations d'envergure suffisante pour assimiler la forte croissance démographique de l'aire urbaine. Les opérations excentrées n'ont pas donné naissance à de nouvelles infrastructures de TCSP*, et si de prime abord l'urbanisation semble mieux coordonnée avec le réseau ferroviaire, la redondance des tracés avec les principaux axes routiers et l'absence de requalification des voies ferrées en service urbain remet partiellement en cause cet espoir de bonne pratique. Le lien densité / TCSP* existe bien, mais c'est la présence d'une urbanisation dense qui a été le préalable nécessaire à l'ouverture de ces lignes, subordonnant ainsi le transport en site propre à l'urbanisation, et non l'inverse comme le Schéma Directeur le préconisait. L'exemple de Blagnac, résumé par R. Marconis (2011), illustre cet état de fait : une fois encore, c'est a posteriori qu'il a fallu résoudre le problème des transports en inscrivant le tracé de la nouvelle ligne dans un territoire qui s'était urbanisé depuis plusieurs décennies en fonction des déplacements en automobile (...) ». 
Figure 1. Toulouse : aire urbaine et InterSCoT

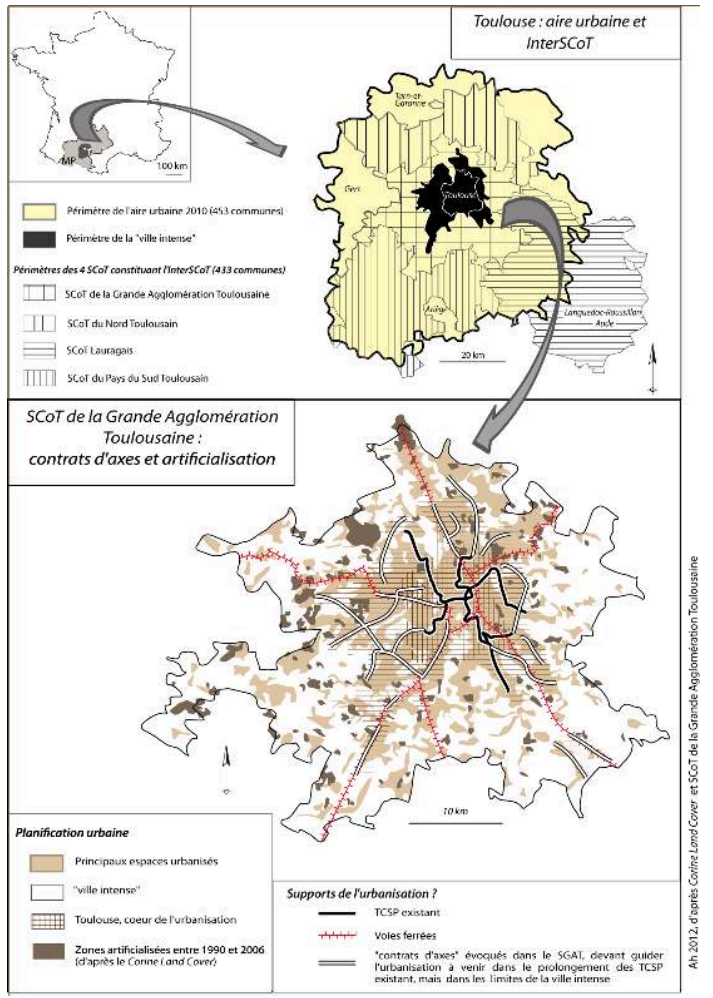

18 Les orientations majeures du Schéma Directeur, telles que l'utilisation du réseau routier secondaire comme support de l'intensification et de la recomposition urbaine, illustrent l'abandon des modes lourds dans les secteurs excentrés. Elles ne participent de l'articulation transports collectifs / urbanisation qu'au prix d'un partage de la voirie intégrant un site propre. Quelques communes de la proche agglomération toulousaine ont fait ce choix, avec toutefois la mise en place d'un bus dans un premier temps (voir infra). Ces solutions nécessitent toutefois un périmètre de gestion des transports collectifs cohérent. Ses définitions complexes et évolutives n'ont certainement pas favorisé l'application des mesures volontaristes : si le Périmètre des Transports Urbains (PTU*) desservi par l'AOT* comptait alors 85 communes, le PDU* en incluait 13 de moins, et jusqu'à la mise en place de l'interSCoT, le Schéma Directeur et ses préconisations se limitaient à 53 communes, délaissant les territoires les plus concernés par l'étalement. La subordination de l'urbanisation aux transports collectifs ne peut dès lors passer que par la sur-densification d'axes déjà suffisamment denses pour avoir justifié l'implantation d'un TCSP*, ou présentant un tel potentiel.

C'est justement le choix mené par l'Allemagne, qui offre aux TCSP* un véritable rôle de vecteurs de l'aménagement urbain. Elle bénéficie du contexte favorable d'une forme urbaine dont les quartiers excentrés et les noyaux secondaires sont denses et bien équipés, et souvent bien reliés. La moindre atteinte portée aux réseaux historiques, et le développement précoce et d'envergure de diverses formes de TCSP* (tramway, tramtrain, S-Bahn, O-Bahn d'Essen...), sont les premiers atouts des agglomérations allemandes, qui bénéficient ainsi de bonnes dessertes jusque dans les banlieues. Ces pôles offrent autant de supports autour desquels orienter la croissance urbaine, en alternative à la suburbanisation. Mais au-delà de cette simplicité apparente, façonnée par des décennies d'aménagement du territoire, certains Länder ont mis en place des 
politiques volontaristes, relayées par les communes. L'exemple de la Rhénanie-DuNord-Westphalie et de Münster $^{9}$ nous permet de dresser un bilan de ces expérimentations.

Le Land de Rhénanie du Nord - Westphalie est particulièrement investi dans la déclinaison régionale de la planification urbaine décidée par l'État fédéral. Après sept années de concertation, il a établi en 1995 puis renforcé en 1998 un programme de subordination des nouvelles constructions du Land aux transports en commun. Les subventions à la construction y sont assujetties à la proximité d'un site propre (moins de $500 \mathrm{~m}$ ) ou d'une gare de $S$-Bahn (1 km maximum), dans la limite de la première périphérie urbaine et sous condition de réaliser de l'habitat collectif ou intermédiaire. L'articulation urbanisme / transports se joue ensuite à l'échelle des communes, qui à l'image de Munster, décident (ou non) de décliner ces initiatives régionales dans le cadre de la planification de l'usage de leurs sols, avec l'aide financière octroyée par le Land. Münster, ville-arrondissement de 280000 habitants (2010) dispose d'un vaste territoire de $303 \mathrm{~km}^{2}$, composé du cœur urbain, d'une première couronne dans la continuité du centre, et d'une dizaine de quartiers excentrés (5 à $10 \mathrm{~km})$, séparés du cœur par une ceinture rurale mais raccordés par le réseau de transports urbains, géré par la ville-arrondissement elle-même. La plupart bénéficient également d'un arrêt sur l'une des 9 lignes de S-Bahn desservant l'agglomération, et qui la relient au vaste bassin de vie d'un million et demi d'habitants qu'elle polarise (Figure 2).

Figure 2. Münsterland : taches urbaines et artificialisation

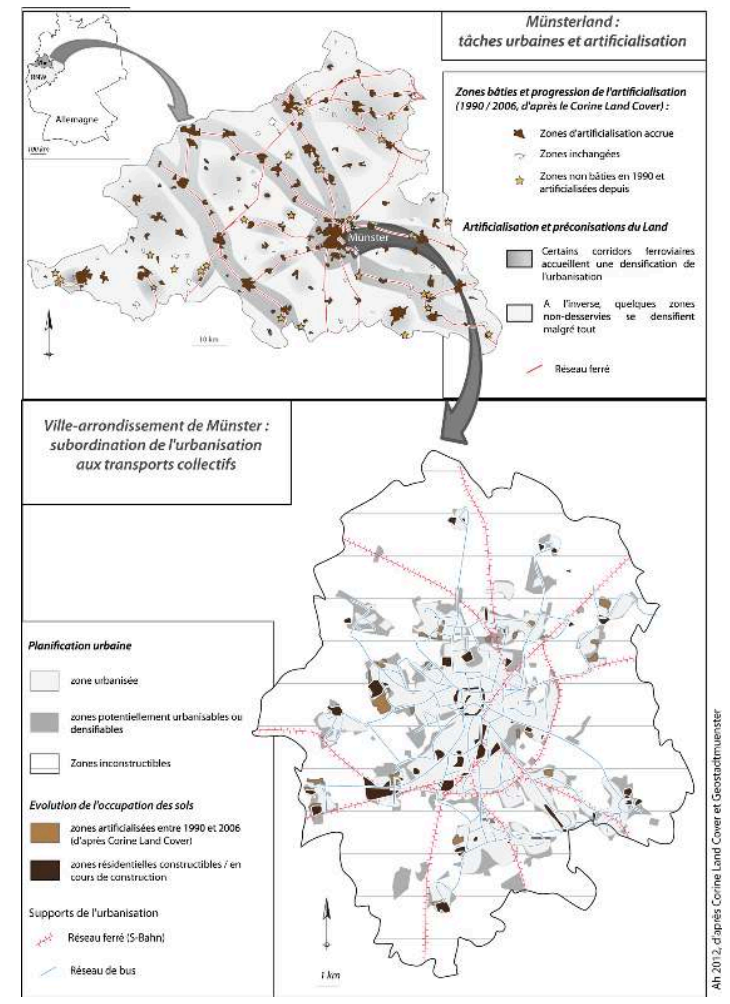

21 La municipalité a donc adopté ses propres critères d'urbanisation, calqués sur ceux du Land. L'évolution de la cité y est planifiée autour de polarités secondaires de 5 à 10000 habitants, bien équipées et desservies par les TCSP*, dont elle densifie friches et « dents creuses ». L'urbanisation est freinée voire gelée dans les autres secteurs, notamment 
dans la couronne rurale. Restreindre l'étalement incontrôlé passe également par le développement d'une offre de logements dense, mais qui réponde aux demandes des familles (plus d'espace et de verdure). Une politique foncière volontariste a alors vu le jour, qui a vu la municipalité procéder à l'acquisition prospective et massive de terrains bien situés ouverts à l'urbanisation. Ces réserves foncières, qui en font la propriétaire de la moitié des surfaces constructibles de la ville, lui permettent de contrôler la spéculation foncière sur ces terrains stratégiques, de subordonner efficacement les nouvelles constructions aux sites propres et d'en contrôler le mode d'urbanisation. Viabilisés et équipés, bien desservis par les transports en commun, ces terrains sont revendus à un prix abordable pour une construction répondant aux normes émises par la municipalité, notamment en termes de densité (petits immeubles ou maisons de ville multifamiliales).

Le bilan de ce programme s'avère favorable sur trois points. En termes quantitatifs, puisque depuis 1990, $75 \%$ des constructions nouvelles dans l'agglomération, soit 17000 logements ont été construits à moins de $800 \mathrm{~m}$ du cœur d'une polarité secondaire, et dans un rayon inférieur à $1,5 \mathrm{~km}$ d'une gare. Par ailleurs, ces contraintes fortes n'ont pas généré de rejet : alors que toutes les villes du Land tendent à voir leur population stagner voire diminuer, celle de Münster augmente : de 260000 habitants en 1990, elle est passée à 280000 au recensement de 2010. Enfin, les comparaisons du Corine Land Cover à l'échelle de la ville montrent la quasi-absence d'atteintes aux couronnes non bâties en 1990 (Figure 2).

La comparaison nous permet de mettre en exergue trois points qui favorisent la réussite des agglomérations subordonnant la croissance urbaine aux TCSP* : d'une part la présence d'une structure urbaine qui, si elle est marquée par la périurbanisation, concentre cet étalement au sein de polarités secondaires suffisamment denses pour permettre leur desserte en TCSP*. L'offre de ces derniers est par ailleurs sans comparaison avec celles des agglomérations françaises, favorisant le report modal vers les transports collectifs. Enfin, le périmètre des AOT* coïncide avec l'échelle fonctionnelle de la ville-arrondissement et du Land, qui sont également celles des politiques de coordination urbanisme / transport.

Deux pays, deux contextes et deux conceptions différentes des TCSP* pour un même objectif ... on voit toutefois apparaître en France et en Allemagne de nouvelles orientations qui pourraient tendre à la convergence des modèles.

\section{De l'inflexion à la convergence des modèles ?}

Les méthodes et premiers résultats semblent opposer France et Allemagne, pourtant liées par un même objectif. Au-delà de Münster, d'autres agglomérations font montre de politiques volontaristes fortes. Ainsi, Munich est un exemple reconnu et souvent imité de compacité urbaine et de limitation de l'étalement urbain ; elle est notamment parvenue à contenir son étalement en dépit de l'accroissement de la population, et à améliorer de $20 \%$ la fréquentation de ses transports collectifs (Jouve 2003) ${ }^{10}$. D'autres agglomérations, telles que Stuttgart ou Rhin-Main, ont mis en œuvre des Verband Region, associations coordonnant à l'échelle métropolitaine les planifications urbaines et de transport. Dans la région métropolitaine Rhin - Neckar, les communes connectées au réseau S-Bahn sont autorisées à croître davantage que les autres (Mandel et al. 2008), tandis qu'à Stuttgart, les critères d'ouverture à l'urbanisation excluent 
notamment les secteurs éloignés de plus de $500 \mathrm{~m}$ d'une gare ou d'un arrêt de TCSP (Wulfhorst 2007, ILS NRW 2001).

Néanmoins, l'articulation entre urbanisme et transports collectifs reste fragile. La coordination des différentes politiques sectorielles peut subir un manque d'entente entre les autorités en charge de l'urbanisme et des transports régionaux. Par ailleurs, dans le cadre de la subsidiarité, l'obtention de résultats à l'échelle nationale nécessite une bonne volonté généralisée en termes de pratiques d'aménagement, ce qui peut constituer un point faible du modèle allemand. Ainsi, dès 2001, l'Institut de recherche pour le développement régional et urbain du Land de la Rhénanie du Nord - Westphalie (ILS-NRW) déplorait que, même dans cette région pourtant souvent citée en modèle, la cohérence transport / urbanisme ne soit pas toujours déclinée à l'échelle des régions urbaines, faute de volonté politique locale: "(...) les GEP ${ }^{11}$ de Rhénanie-Westphalie contiennent de nombreuses zones d'urbanisation sans desserte ferrée (...) et dans celles qui sont desservies, la concentration autour des arrêts n'est pas marquante ». Par ailleurs, il pointait du doigt le manque de précision de ces documents : «il n'existe pas à l'échelle du LEP ${ }^{12}$ ni à celle des GEP*, d'objectifs quantifiés ou des critères précis concernant la localisation, la mixité des fonctions, la densité ou la desserte en transports publics, qui pourraient limiter un développement déconnecté du réseau ferré».

Face à des plans régionaux peu précis, la subsidiarité offre aux communes comme aux Länder la souveraineté d'appliquer avec plus ou moins de rigueur ces principes dans leurs propres documents, ou de céder à l'impopularité grandissante des mesures. En effet, en dépit d'une importante communication et d'une bonne gouvernance, les communes qui se voient bloquées dans leur développement économique et démographique manifestent leur mécontentement. Or le principe de " contre-courant " (Gegenstromprinzip) implique que la politique de la ville soit définie à la fois de façon descendante -de l'État au Land et aux communes - mais aussi de façon ascendante - des communes vers le Land et l'échelon fédéral. Dès lors, même si la politique générale d'articulation urbanisme / TCSP* n'est pas abandonnée, l'influence mutuelle des planifications locales et régionales favorise localement des assouplissements dans son application, visant à atténuer ces tensions.

Côté français, la contraction urbaine concerne essentiellement de petites agglomérations mal situées - le contexte est davantage encore à la croissance urbaine et à l'étalement (Fol et Cunningham-Sabot 2010). L'échelle de réflexion pour contraindre ce dernier s'inscrit désormais dans le territoire fonctionnel des régions urbaines, jusqu'alors sans pouvoir politique ni administratif, à l'intérieur duquel la coordination urbanisme / transport doit pouvoir se dégager - à la fois parce qu'elle est exigée par la loi, mais aussi parce que les périmètres d'intervention des politiques sectorielles peuvent enfin être mis en cohérence. Ainsi, le SCoT* de la Grande Agglomération Toulousaine (SGAT) recoupera le périmètre de 117 communes du PDU* approuvé en 2011, qui devrait favoriser la desserte des espaces périurbains. Avec le $\mathrm{SCoT}^{*}$ s'impose également l'idée d'une urbanisation subordonnée aux transports collectifs, qui devront se poser en vecteurs de projets d'aménagement. Le rapport de présentation du SGAT* (juillet 2010) prévoit que «toute ouverture à l'urbanisation ou projet de renouvellement urbain d'ampleur doit s'accompagner d'une desserte en transports collectifs adaptée ». Par ailleurs, "l'ouverture à l'urbanisation de nouvelles zones dans les PLU devra concerner les secteurs déjà desservis par les transports en commun ou les terrains en continuité immédiate du cour de ville ou de villages ». 

(métro ligne A), une autre de 145 ha à Borderouge (ligne B) constituent les premiers signes d'une densification autour des terminus du métro. Ils participent également du principe de résilience urbaine, qui prône une recomposition de la ville sur la ville et la réurbanisation des espaces en déshérence, un précepte également partagé avec l'urbanisme allemand. En Allemagne toutefois, cette densification accompagne le renforcement du polycentrisme ancien, qui offre au final plusieurs pôles de densités globalement homogènes (Kauffmann et Jemelin 2010) et une répartition des équipements de proximité sur l'agglomération. Cette structure polycentrique en réseau présente les meilleures opportunités pour le développement des TCSP* et la contrainte de la croissance urbaine. concentration autour de polarités secondaires, mais s'oriente souvent vers la densification de la «ville intense $»^{13}$ - pourtant déjà suffisamment densifiée pour justifier une desserte en TCSP*. Si le réemploi de vastes friches participe de la reconquête d'un secteur en déshérence et de la restructuration d'un tissu urbain ainsi « recousu »- requalification de l'ancien site industriel de la Cartoucherie par exemple le risque demeure de pousser la densification des espaces centraux au-delà des capacités de la ville à la gérer, conduisant à s'interroger sur l'efficacité d'un tel accroissement du différentiel entre un espace central particulièrement compact et une banlieue étalée. Divers auteurs ont déjà soulevé une moindre efficience des mobilités dans une ville trop resserrée, le rejet potentiel d'une forme urbaine dense non désirée (Breheny 1997), ou encore le renforcement de la gentrification lorsque la raréfaction de l'espace disponible et les opérations de renouvellement urbain qui accompagnent les nouvelles infrastructures contraignent les coûts fonciers à la hausse, et les plus économiquement fragiles au départ vers les couronnes périurbaines (Pouyanne 2004).

31 La loi Grenelle 2 et les SCoT* qui s'en inspirent mettent toutefois également l'accent sur des opérations destinées à densifier des corridors de peuplement raccordant des polarités secondaires, en vue de leur desserte par un TCSP*. Avec ces «contrats d'axes ", la densification gagne des espaces moins centraux. Le Grand Toulouse a engagé la réflexion depuis une dizaine d'années, et envisage la négociation de tels contrats entre l'AOT* et les communes desservies dans l'objectif de renforcer la cohérence urbanisme / transport et d'étendre le réseau de TCSP*. Le manque de densité des banlieues péricentrales les prédispose en effet à l'accueil de simples bus, alternatives qui souffrent d'un déficit d'image altérant leur crédibilité face aux modes motorisés individuels. Pour favoriser l'émergence d'un projet de TCSP* ou l'amélioration d'une desserte ferrée sur un territoire péricentral, communes et intercommunalités concernées s'engagent à en densifier la zone d'influence afin de garantir une fréquentation satisfaisante à la future infrastructure. Le SGAT* envisage ainsi 27 contrats d'axes potentiels, accompagnant une vingtaine de TCSP* inscrits au PDU*. Implantés dans le prolongement des lignes existantes, ces potentiels contrats d'axes demeurent encore circonscrits aux limites de la «ville intense» (Figure 1). Ils devraient faire émerger des centralités secondaires dans la première couronne, combinant densité urbaine et mixité des fonctions, et en faire de réels axes structurants. En cela, on peut effectivement constater un infléchissement du modèle français, dans la droite ligne du Nouvel Urbanisme et des espoirs de durabilité qui s'y rapportent.

Revue Géographique de l'Est, vol. 52 / 1-2 | 2012 

semble prise à l'envers: l'objectif de la densification attendue réside dans le financement du TCSP* et dans un niveau de fréquentation à venir satisfaisant, davantage que dans l'émergence d'une forme urbaine raisonnée. Certes, enfin, le projet de TCSP* précède la densification urbaine, et ne s'inscrit plus longuement en aval. C'est un progrès. Mais il n'en demeure pas moins qu'il s'agit d'une subordination du TCSP* à l'urbanisation - si la densification échoue, la liaison peut être abandonnée. Les 7,8 kmdont $4 \mathrm{~km}$ en site propre - reliant le terminus de métro Ramonville à Castanet par l'exRN 113 illustrent ce constat. Ce projet a constitué le ballon d'essai des contrats d'axe sur l'agglomération toulousaine ${ }^{14}$. Les 38 habitants/ha dans le périmètre des $400 \mathrm{~m}$ autour de l'axe n'étant guère favorables à la réalisation d'un TCSP*, un bus a été mis en place et des scénarii de densification ont été établis. A terme, si l'évolution de la typologie urbaine le permet, le mode sera adapté pour un TCSP* plus efficace et sur l'intégralité du tracé. Le PDU* impose en effet le choix du mode aux densités et typologies urbaines - le seuil recommandé pour un BHNS est de 140 habitants, 50 logements et 30 emplois/ha en moyenne ${ }^{15}$.

En dépit de ces quelques réserves, les mesures issues des SCoT* tels que les contrats d'axe offrent l'espoir d'une réelle avancée dans la question de la subordination de l'urbanisme aux TCSP*. Cette démarche, encore expérimentale, a déjà séduit quelques agglomérations qui mettent en place de telles opérations, parfois sous des vocables différents - les DIVAT de l'agglomération lilloise par exemple - et à aide de supports variés : Grenoble s'appuie ainsi que une nouvelle ligne de tramway, Nîmes et Alès sur l'amélioration de l'offre ferroviaire, l'île-de-France sur le prolongement d'une ligne de métro. Trop récents pour offrir un véritable recul sur ces démarches et leurs réels potentiels, ils délivrent toutefois l'image de programmes à même d'apporter de la cohérence dans un cadre qui en manque parfois cruellement. Les démarches préparatoires illustrent leur principale qualité, la capacité de fédérer autour de projets très précis des synergies et des acteurs différents, intégré dans un périmètre opérationnel concret et homogénéisé. Les premiers retours sur expérience insistent également sur l'impact pédagogique marqué des négociations auprès des élus locaux. On peut toutefois soulever et regretter que, jusqu'à présent, ces contrats n'impliquent que les acteurs publics et omettent les interlocuteurs privés, pourtant importants facteurs de croissance urbaine et de densification tant de l'emploi que de l'habitat. Par ailleurs, la qualité purement contractuelle de ces contrats d'axe peut faire craindre un manquement aux engagements pris s'ils ne sont pas, à terme, intégrés aux outils de planification, les seuls à même de leur concéder un caractère d'obligation légale qui pourrait assurer leur réelle mise en pratique.

34 Dans tous les cas, ces projets, s'ils ne sont pas accompagnés de mesures complémentaires et de réflexions sur la forme urbaine dense, risquent de rester insuffisamment efficaces: l'aire urbaine de Toulouse accueille chaque année 20000 nouveaux habitants, et le SDAG s'attend à l'arrivée de 250 à 300000 habitants supplémentaires d'ici 2030 -l'INSEE en prévoit 470000 pour 2042. Tous ne pourront ni ne voudront intégrer ces espaces péricentraux densifiés, même bien desservis, et les seuls contrats d'axe ne pourront contrer les menaces d'étalement urbain supplémentaire. Le SDAG* lui-même, qui espère pourtant avec optimisme concentrer $80 \%$ des nouveaux arrivants dans la ville intense, prévoit également encore une artificialisation des terres agricoles et naturelles de l'ordre de 340 ha/an, soit une 
augmentation de $25 \%$ de sa superficie actuellement urbanisée. L'étalement va donc se poursuivre au-delà du périmètre des transports urbains, et nécessiter de nouvelles mesures, tant pour ces zones excentrées que pour convaincre les néo-arrivants de ne pas grossir le flux des prétendants aux $340 \mathrm{ha} /$ an de nouvelles zones périurbaines.

\section{Conclusion}

La mise en œuvre de mesures législatives déclinées dans des documents de planification et d'urbanisme ambitieux est un préalable nécessaire à l'émergence d'une coordination urbanisme / transports collectifs en site propre, mais qui pourrait ne pas être suffisante. L'expérience passée de l'agglomération toulousaine et l'exemple de Münster illustrent la nécessité d'une volonté politique forte et sur le long terme (la tradition aménagiste allemande s'étire sur plus d'un siècle), et l'influence d'un cadre fonctionnel et urbain dont l'évolution peut s'avérer longue. Cette réflexion ouvre également la question des choix opérés pour ces espaces bien situés, car l'efficacité de cette politique d'articulation repose également sur l'attractivité de la forme urbaine proposée.

36 La réussite des quartiers densifiés allemands repose en partie sur cette combinaison de bonne desserte, de mixité fonctionnelle, mais également d'aménité. Si la structure polyconcentrique en réseau demeure loin de nos traditions urbaines et ne sera pas recréée aisément, le choix de formes densifiées acceptables sera fondamental sous peine de voir les familles délaisser ces secteurs comme elles délaissent les couronnes de proche banlieue, pourtant bien équipées et desservies. Proposer une alternative crédible et acceptée au pavillon encerclé de son jardin fait aussi partie des défis à ne pas occulter dans la réflexion sur la subordination de la forme urbaine aux TCSP*.

Polycentrisme à l'allemande, renforcement de cœurs urbains raccordés par des TCSP*, nouvelles centralités, les modalités de (re)composition de ces quartiers prennent diverses directions, à la recherche d'un consensus sur le meilleur choix possible pour réconcilier ville et mobilités. Pourtant, la fenêtre de tir est étroite. D'autres stratégies automobiles naissent pour pallier la fin du pétrole, les TCSP* lourds semblent ralentir, la spéculation s'empare des terrains autour des gares et des friches ... il y urgence, pour ne pas laisser passer cette opportunité de mieux aménager une ville «durablement » mobile.

BIBLIOGRAPHIE

BREHENY M., 1997, “Urban compaction : feasible and acceptable ?", CitiesVol. 14, No. 4, pp. 209-217

FOL S. et CUNNINGHAM-SABOT E., 2010, « Déclin urbain » et Shrinking Cities : une évaluation critique des approches de la décroissance urbaine ", Annales de géographie, 2010/4 n 674, p. 359-383 
ILS NRW, 2001, Mobilité et urbanisme en Allemagne ; promouvoir l'urbanisation autour des dessertes ferrées et des TCSP : la politique du Land de Rhénanie du Nord-Westphalie (T1) et Des Politiques locales exemplaires (T2), Dortmund, ILS NRW, Ademe, Predit 2, 64 p.

JOUVE B., 2003, Les politiques de déplacements urbains en Europe, l'innovation en question dans cinq villes européennes, Paris, L'Harmattan, coll. Logiques Politiques, 191 p.

KAUFMANN V. et JEMELIN Ch., 2003, « Articulation entre urbanisme et transports : quelles marges de manœuvre? ", Revue internationale des sciences sociales, $\mathrm{n}^{\circ} 176$ (2003/2), pp. 329-340.

LAISNEY F., 2011, Atlas du tramway dans les villes françaises, Paris, Éditions Recherches, 422 p.

MANCEBO F., 2008, « Une périurbanisation durable : des écueils aux opportunités », in VALLAT C. (dir) Pérennité urbaine ou la ville par-delà ses métamorphoses vol.2 Turbulences, L'Harmattan, pp. 21 32.

MARCONIS R. (dir.), 2011, Toulouse, les territoires du tramway, Quand les transports repensent l'agglomération, Toulouse, $140 \mathrm{p}$.

MANDEL (K.) et al., 2008, « Mannheim, à la découverte de la mécanique de la ville rhénane », Transports Urbains, $\mathrm{n}^{\circ} 113$, juin 2008, pp. 27 - 30

NEWMAN P. et KENWORTHY JR, 1998, Sustainability and cities - overcoming automobile dependence, Washington DC, Island Press, $450 \mathrm{p}$.

OCDE, 1999, La politique de la ville en Allemagne, vers un développement urbain durable, Paris, Les éditions de l'OCDE, coll. Développement territorial, $106 \mathrm{p}$.

POUYANNE G., 2004, « Des avantages comparatifs de la ville compacte à l'interaction forme urbaine - mobilité. Méthodologie, premiers résultats ", Les Cahiers scientifiques du Transport $\mathrm{n}^{\circ}$ 45/2004 - pp. 49-82.

WIEL M., 2010, Étalement urbain et mobilité, Paris, La Documentation Française, coll. Transports, recherche, innovation, $86 \mathrm{p}$.

WULFHORST G., 2007, «L'intégration entre politiques territoriales et politiques des transports en Allemagne : l'exemple de la région urbaine de Stuttgart », Flux, 2007/3 n 69, p. 63-72.

\section{Table des sigles :}

AOT : Autorité Organisatrice de Transports.

GEP : Gebietsentwicklungsplan - Plan de développement des régions urbaines.

LAURE : Loi cadre n $96-1236$ du 30 décembre 1996 sur l'Air et l'Utilisation Rationnelle de l'Énergie.

LEP : Landesentwicklungsplan - Plan de développement régional(échelle du Land).

Loi SRU : Loi n²000-1208 du 13 décembre 2000 relative à la solidarité et au renouvellement urbains.

LOTI : Loi n 82-1153 du 30 décembre 1982 d'Orientation des Transports Intérieurs.

PDU : Plan de Déplacement Urbain.

PTU : Périmètre des Transports Urbains.

RER : Réseau Express Régional.

SCoT : Schémas de Cohérence Territoriale. 
SGAT : SCoT de la Grande Agglomération Toulousaine.

TCSP : Transport Collectif en Site Propre (variante : Transport en Commun en Site Propre).

VAL : Véhicule Automatique Léger (à l'origine Villeneuve-D’ascq - Lille) ; métro sur pneu automatique.

ZAC : Zone d'Aménagement Concerté.

\section{NOTES}

1. ... ou " pic pétrolier ». Terminologie issue de l'exploitation pétrolière, désignant le sommet de la courbe de production d'un puits. Cette expression est couramment employée pour désigner, à l'échelle mondiale, le moment où la production cessera de croître avant de décroître en raison de l'épuisement des réserves.

2. Les mots suivis d'un astérisque renvoient à la table des sigles à la fin de l'article

3. Code de l'Urbanisme, article 122-1, version abrogée du 13 janvier 2011

4. Respectivement «Plan Local d'Urbanisme » et « Plan de Déplacements Urbains »

5. Ministère de l'Écologie, du Développement Durable, des Transports et du Logement

6. Bundesministeriums für Raumordnung, Bauwesen und Städtebau (1993), Zukunft Stadt 2000, Bonn.

7. Son évolution rapide et l'extension de l'urbanisation a justifié l'évolution de sa définition par l'INSEE : en octobre 2011, elle recouvre 453 communes et 1,2 millions d'habitants.

8. cf lignes « attrape-tout » de Pierre ZEMBRI, dans ce numéro : La conception des TCSP en France : des tracés problématiques?

9. Voir notamment les différentes interventions et publications de Patricia GOUT, par exemple ILS NRW (2001)

10. On pourra également se référer à la publication en ligne de l'ADEME et d'Energy-Cities (2009), "Garder Munich compacte, urbaine et verte », 7 p. (accessible http://www.energycities.eu/db/munich_578_fr.pdf, page consultée en mars 2012).

11. GEP :Gebietsentwicklungsplan - Plan de développement des régionsurbaines

12. LEP : Landesentwicklungsplan - Plan de développement régional(échelle du Land)

13. La ville intense "désigne le cour de l'agglomération soit la ville centre et les communes des pôles secondaires (en première couronne majoritairement). La ville intense constitue le lieu privilégié d'accueil de la population, avec un objectif élevé de densité urbaine en lien avec le niveau de service des réseaux de transport en commun ». Définition Tisséo, "des Idées pour mieux bouger », Dossier de synthèse, projet de révision PDU.

14. La ligne ayant été réalisée au cours de l'étude, le contrat d'axe ne sera finalement pas signé, mais il a permis de sensibiliser les élus à ce concept.

15. SCoT de la Grande Agglomération Toulousaine, Document d'Orientations Générales, projet arrêté juillet 2010. 


\section{RÉSUMÉS}

Face à l'étalement urbain galopant et à l'artificialisation de leurs territoires, France et Allemagne se sont lancées dans des politiques visant à contraindre la croissance urbaine au long des dessertes de transports en commun en site propre. Partageant les mêmes objectifs, ces politiques s'inscrivent toutefois dans des cadres structurels et urbains différents, qui influent sur leur mise en œuvre et leur efficacité. En Allemagne, la structure polynucléaire historique renforcée par l'aménagement du territoire, associe polarités secondaires denses et mixtes fonctionnellement à une bonne desserte ferroviaire, ce qui a favorisé l'orientation de la périurbanisation dans ces secteurs. Le Land de Rhénanie du Nord-Westphalie a obtenu quelques bons résultats dans ce domaine, notamment dans la ville-arrondissement de Münster. A l'inverse, les premières mesures issues du Schéma Directeur de Toulouse ne se sont pas avérées aussi concluantes, la progression de la tache urbaine restant globalement déconnectée du réseau de Transports Collectifs en site propre (TCSP). La réflexion à une nouvelle échelle fonctionnelle englobant les espaces périurbains que permettent les SCOT devrait favoriser l'émergence de nouvelles pratiques telles que la densification des secteurs bien desservis et la négociation de " contrats d'axes » qui devraient accroître le réseau de TCSP et en assurer la fréquentation.

Facing rampant urban sprawl and land consumption, France and Germany have initiated policies in order to constrain urban growth along reserved lanes public transport network. Sharing the same objectives, these policies depend on different urban and politic frameworks that affect their implementation and effectiveness. In Germany, the historic polynuclear structure was reinforced by the national planning and development. It combines secondary dense and mixed polarities with a high level rail service, which has promoted the orientation of suburbanization in these areas. The Land of North Rhine-Westphalia has achieved some good results, particularly in the city-district of Münster. Conversely, the first steps of the Master Plan of Toulouse have not been as conclusive. The progress of urban sprawl remains disconnected from the public transport network. The "SCoT" (New French urban planning document) encourages considering urban planning at a new functional scale encompassing the suburban areas. It should encourage the emergence of new practices such as the densification of areas well served by public transport and the negotiation of "corridors contracts" which should increase the size of the network and ensure its attendance.

\section{INDEX}

Keywords : coordination of land use and transport policies, urban sprawl, urban planning, corridors contract, reserved lane public transport networks

Mots-clés : coordination urbanisme / transports, étalement urbain, planification urbaine, Contrats d'axes, TCSP, SCoT

\section{AUTEUR}

\section{ANNE HECKER}

Université de Nancy 2, CERPAAnne.Hecker[chez]univ-nancy2.fr 